\section{High selectivity UWB bandpass filter with controllable bandwidth of dual notch bands}

\author{
M. Mirzaee, S. Noghanian and B.S. Virdee
}

A technique to implement and control the bandwidth of notch bands in an ultra-wideband (UWB) bandpass filter (BPF) based on signal superposition is described. The filter is realised by employing two wideband BPFs in a parallel configuration to establish two signal paths between input/output ports. The proposed technique can substantially increase the bandwidth of the notch band by $\sim 370 \%$ in comparison with the notch-band filter designs based on transversal signal interaction. The proposed filter exhibits a quasi-elliptical response over the span of the UWB spectrum defined by the Federal Communication Commission. The compact filter design has a size of $88.92 \mathrm{~mm}^{2}$

Introduction: Ultra-wideband (UWB) technology offers great potential for high-speed data, voice and video transferring over a wide frequency span with low transmission power. This low-cost technology is suitable for emerging short-range wireless applications. Bandpass filters (BPFs) required for integration in UWB systems must provide low insertion loss, high selectivity and flat group delay to minimise distortion of UWB signals. In addition, to reduce the size of the transceiver and efficiently utilise the UWB spectrum with signals from other services, many UWB BPFs with single or multiple notch bands have been explored over the past few years based on the following techniques: transversal signal interaction [1-3], composite right/left-handed transmission-line unit cells [4], multilayer LCP technology [5], the triple-mode stepped impedance resonator (SIR) [6], the E-shaped resonator [7] and defected ground structures (DGSs) [8]. From a practical point of view, DGS and multilayer LCP filter designs are complex and have a high manufacturing cost.

In this Letter, a novel approach is introduced to implement and control the bandwidth of the notch bands within the UWB spectrum. The proposed technique of creating notch bands is based on signal superposition for different transmission paths. The technique enables the bandwidth of the notch band to be increased by $\sim 370 \%$ in comparison with the techniques based on transversal signal interaction concepts [2]

Methodology: Fig. $1 a$ shows the structure of the proposed UWB filter. It comprises two transmission paths, each of which includes a folded trisection SIR that is electromagnetically coupled with the input/output lines to enable signal transmission. The input signal propagates from port 1 to port 2 along paths 1 and 2 through the wideband filters, referred to here as filter-A and filter-B. The design of the wideband BPFs is described in [2]. To have more control over the resonant modes, trisection folded SIRs are employed in the filter design. Fig. $1 b$ shows the equivalent circuit model of the proposed resonator. Since the SIR is symmetrical in the $P-Q$-plane, its resonance condition can be analysed using even-mode and odd-mode analyses [2]. The approximate equivalent circuits for odd-mode and even-mode excitations are illustrated in Fig. $1 b$. The resonance conditions for even-mode and odd-mode are defined by

$$
Y_{3} \tan \theta_{3} \tan \theta_{2}-Y_{2}=0
$$

$Y_{2}\left(Y_{1} \tan \theta_{1}+2 Y_{2} \tan \theta_{2}\right)+Y_{3} \tan \theta_{3}\left(2 Y_{2}-Y_{1} \tan \theta_{1} \tan \theta_{2}\right)=0$

In [2], the notch bands are generated based on transversal signal interaction. Although two notch bands can be created within the UWB spectrum by forcing the signal propagating on paths 1 and 2 to cancel at the output port, the problem with this method is that it is not capable of producing wide notch bands, especially in the case of the second notch band. To achieve a wider and a more controllable second notch band, the approach proposed here is based on the superposition of signals of the individual BPFs. In this case, the bandwidth of the second notch band can be controlled by changing the lengths $L_{1}+d_{1}$ and $L_{2}+d_{2}$ corresponding to filter-A and filter-B, respectively. Figs. $2 a$ and $b$ shows the individual $s_{21}$ and phase response of the filter-A and filter-B, respectively. The first notch band at about $5.2 \mathrm{GHz}$ is generated based on transversal signal interaction as discussed in [1]. In this region, the two wideband BPFs have equal $S_{21}$ magnitude (see Fig. $2 a$ ) and a phase difference of about $180^{\circ}$ (see Fig. $2 b$ ) as predicted by the transversal signal interaction concept. It can be observed from Fig. $2 a$ that there is a gap of about $8-10 \mathrm{GHz}$ between the $S_{21}$ of filter-A and filter-B which contributes to the creation of a wide second notch band due to the superposition of signals in the final filter structure.

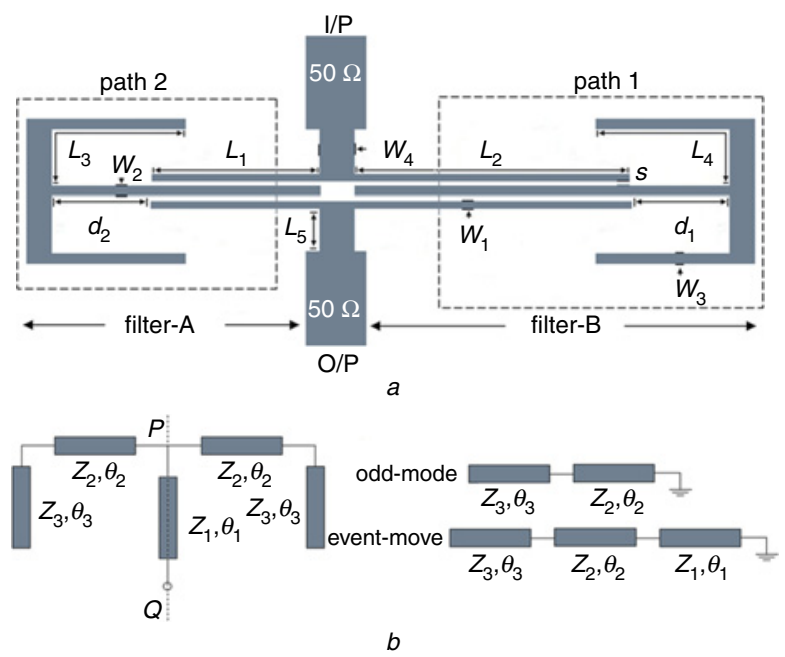

Fig. 1 Configuration of proposed UWB filter, and equivalent circuit model (odd-mode and even-mode) of folded tri-section SIR

$a$ Configuration of proposed UWB filter

$L_{1}=6.9, d_{1}=2, L_{2}=13.4, d_{2}=0.1, L_{3}=4.6, L_{4}=5.4, L_{5}=0.5, W_{1}=0.1, W_{2}=$

$0.2, W_{3}=0.3, W_{4}=1, W_{5}=0.7$ and $S=0.12$ (dimensions in millimetres)

$b$ Equivalent circuit model (odd-mode and even-mode) of folded tri-section SIR
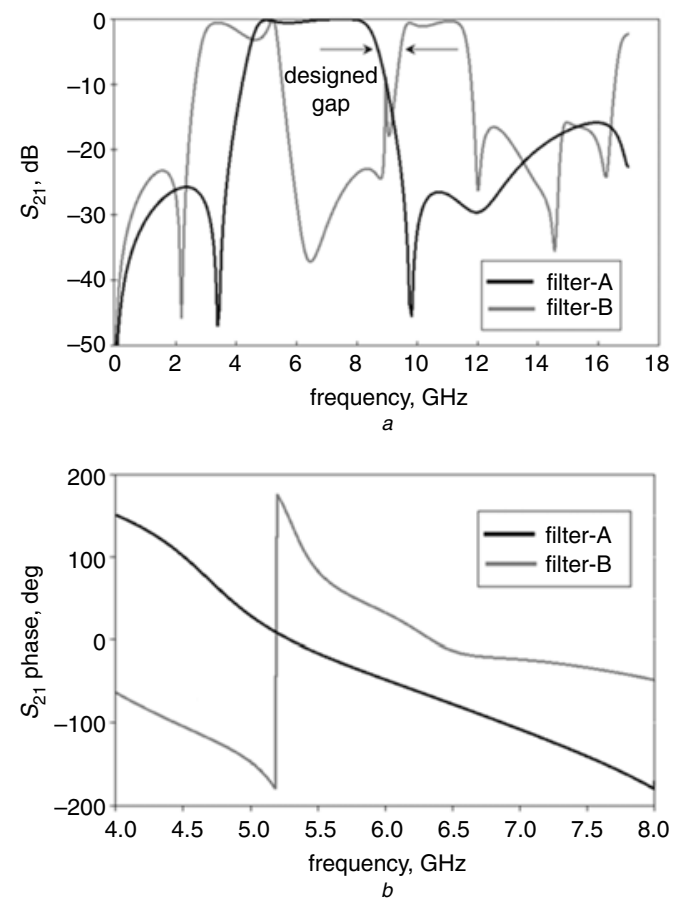

Fig. 2 Simulated insertion loss of individual wideband BPFs in Fig. 1a, and phase response of filter- $A$ and filter- $B$

a Simulated insertion loss

$b$ Phase response

Simulated and measured results: The prototype of the proposed filter design was fabricated on a commercial dielectric substrate with a thickness of 32 mil and a dielectric constant of 3.38. The actual size of the filter is $24.7 \times 3.6 \mathrm{~mm}^{2}$, which is $\sim 6 \%$ more compact in comparison with $[1,2]$. Fig. $3 a$ shows the photograph of the fabricated filter. Figs. $3 b$ and $c$ show the simulated and measured $S$-parameters $\left(S_{21}\right.$ and $S_{11}$ ) and the group delay of the proposed filter, respectively. These results confirm that the proposed filter exhibits an UWB response from 2.96 to $11.75 \mathrm{GHz}$, with a corresponding fractional bandwidth (FBW) of $119.5 \%$ for a return loss $>10 \mathrm{~dB}$. The measured insertion loss is better than $1.19 \mathrm{~dB}$. The first and second notch bands are from 
4.97 to 5.37 and 8.79 to $9.20 \mathrm{GHz}$, respectively. The bandwidth of the second notch band has been increased by about $370 \%$ in comparison with the design in [2]. Moreover, the filter has an almost flat group delay with maximum variation which is $<0.3 \mathrm{~ns}$ except at the notch bands. Table 1 compares the proposed filter with some of the best published UWB filters in terms of size, FBW, notch FBW and location of the notch bands.
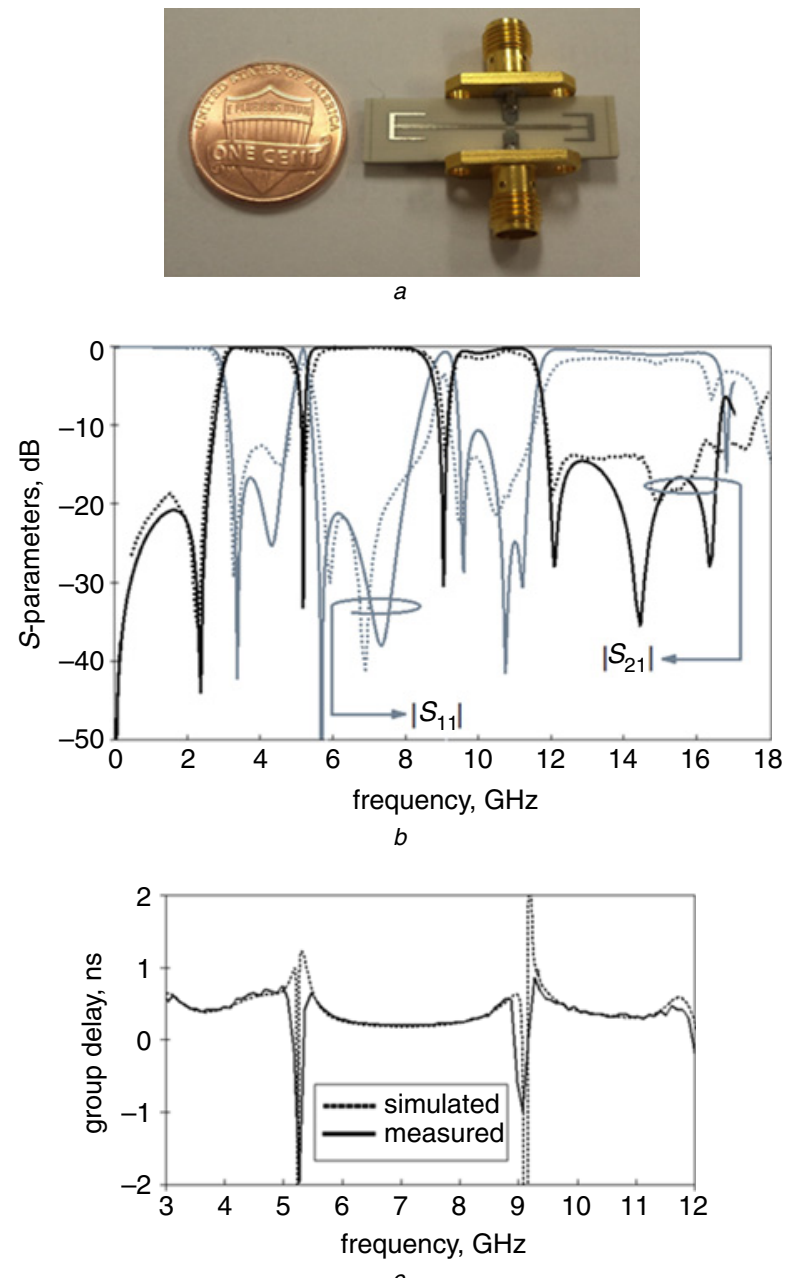

Fig. 3 Photograph of fabricated filter; measured and simulated S-parameters of proposed filter; group delay

$a$ Photograph of fabricated filter

$b$ Measured (dotted) and simulated (solid) $S$-parameters of proposed filter $c$ Group delay

Table 1: Comparison of proposed filter with reported filters

\begin{tabular}{|c|c|c|c|c|}
\hline Ref. & Size $\left(\mathrm{mm}^{2}\right)$ & FBW $(\%)$ & $f$ notch $(\mathrm{GHz})$ & Notch FBW (\%) \\
\hline$[1]$ & 94 & 123.2 & 5.2 & 8.8 \\
\hline$[2]$ & 94 & 123.2 & $5.21 / 9$ & $7.73 / 0.96$ \\
\hline$[3]$ & 127.90 & 101.9 & 5.2 & 3.8 \\
\hline$[4]$ & 78.80 & 114.5 & 5.7 & 1.73 \\
\hline$[5]$ & 405.70 & 106.3 & $5.4 / 5.98 / 6.76$ & $1.11 / 1.34 / 2.95$ \\
\hline$[6]$ & 612 & 118.8 & $5.2 / 5.85 / 8$ & $3.6 / 3.6 / 4.1$ \\
\hline$[7]$ & 296.40 & 108.2 & $5.9 / 8$ & $4.6 / 3$ \\
\hline$[8]$ & 480 & 118 & $5.3 / 7.8$ & $10.5 / 11.02$ \\
\hline This work & 88.92 & 119.5 & $5.2 / 8.9$ & $7.73 / 5.19$ \\
\hline
\end{tabular}

Conclusion: A novel technique has been described to implement and control the bandwidth of the notch bands in a UWB BPF design based on the signal superposition approach. The quasi-elliptical UWB filter exhibits sharp dual notch bands. The bandwidth of the second notch band can be increased by $\sim 370 \%$ in comparison with the notch band created using transversal signal interaction. The proposed UWB filter exhibits excellent insertion loss, return loss and group delay performances. In addition, the filter has a compact size of $88.92 \mathrm{~mm}^{2}$.

(C) The Institution of Engineering and Technology 2014 25 June 2014

doi: $10.1049 / \mathrm{el} .2014 .2336$

One or more of the Figures in this Letter are available in colour online.

M. Mirzaee and S. Noghanian (Electrical Engineering Department, College of Engineering and Mines, University of North Dakota, Grand Forks, ND 58202-7165, USA)

E-mail: milad.mirzaee@my.und.edu

B.S. Virdee (Faculty of Life Sciences and Computing, Centre for Communications Technology, London Metropolitan University, London, United Kingdom)

\section{References}

1 Mirzaee, M., and Virdee, B.S.: 'UWB bandpass filter with notch-band based on transversal signal-interaction concepts', Electron. Lett., 2013, 49, (6), pp. 399-401, doi: 10.1049/el.2012.4203

2 Mirzaee, M., Virdee, B.S., and Noghanian, S.: 'Compact ultra-wideband bandpass filter with variable notch characteristics based on transversal signal-interaction concepts', Int. J. RF Microw. Comput. Aided Eng., 2014, 24, (5), pp. 549-559, doi: 10.1002/mmce.20798

3 Mirzaee, M.: 'A novel small ultra-wideband bandpass filter including narrow notched band utilizing folded T-shaped stepped impedance resonator', Prog. Electromagn. Res. C, 2011, 22, pp. 85-96, doi: 10.2528/PIERC11040811

4 Ahmed, K.U., and Virdee, B.S.: 'Ultra-wideband bandpass filter based on composite right/left handed transmission-line unit-cell', IEEE Trans. Microw. Theory Tech., 2013, 61, (2), pp. 782-788, doi: 10.1109/TMTT.2012.2231432

5 Hao, Z.-C., Hong, J.-S., Parry, J.P., and Hand, D.P.: 'Ultra-wideband bandpass filter with multiple notch bands using nonuniform periodical slotted ground structure', IEEE Trans. Microw. Theory Tech., 2009, 57, (12), pp. 3080-3088, doi: 10.1109/TMTT.2009.2034230

6 Wei, F., Li, W.T., Shi, X.-W., and Huang, Q.-L.: 'Compact UWB bandpass filter with triple-notched bands using triple-mode stepped impedance resonator', IEEE Microw. Wirel. Compon. Lett., 2012, 22, (10), pp. 512-514, doi: 10.1109/LMWC.2012.2215845

7 Zhao, J., Wang, J., Zhang, G., and Li, J.-L.: 'Compact microstrip UWB bandpass filter with dual notched bands using E-shaped resonator', IEEE Microw. Wirel. Compon. Lett., 2013, 23, (12), pp. 638-640, doi: 10.1109/LMWC.2013.2283873

8 Song, Y., Yang, G.-M., and Geyi, W.: 'Compact UWB bandpass filter with dual notched bands using defected ground structures', IEEE Microw. Wirel. Compon. Lett., 2014, 24, (4), pp. 230-232, doi: 10.1109/LMWC.2013.2296291 